$0.49(0.30-0.80), p=0.004$, respectively], while those with a concurrent STI $[1.69(1.15-2.49), p=0.007]$, and those presenting with multiple infection sites [2.54(1.62-4.00), $\mathrm{p}<0.001$ ] were more likely to be culture-confirmed.

Conclusion Not all NAAT-positive attendees were culture-confirmed, but this may be because culture was either unsuccessful or not routinely performed among asymptomatic attendees. All NAAT-positive patients should be cultured before treatment, as routine culture confirmation is essential to ensure representative monitoring of trends in antimicrobial resistance to inform decisions regarding treatment guidelines for gonorrhoea.

\section{P3.285 DIAGNOSTIC AND TREATMENT UNCERTAINTIES: EPIDEMIOLOGICAL RISK FACTORS FOR NAAT POSITIVE BUT CULTURE NEGATIVE GONORRHOEA CASES IN STOCKHOLM, SWEDEN}

doi:10.1136/sextrans-2013-051184.0741

R Vyth, A Leval, B Eriksson, M Hergens. Dept. of Communicable Disease Control and Prevention, Stockholm County, Stockholm, Sweden

Background The advent of gene amplification testing methods for Neisseria gonorrhoea has lead to a higher prevalence of gonorrhoea testing in the population. Various methods for Nucleic Acid Amplification Tests (NAAT) are used, often with high specificity. The sensitivity of culture is substantially lower than NAAT. Before treatment is initiated, antibiotic sensitivity of the isolate should be determined using culture-based methods. A considerable proportion of cases positive with NAAT cannot be verified by culture and hence it is not possible to verify the diagnosis or determine antibiotic sensitivity. Uncertainty in diagnostics and treatment of NAAT positive, culture negative gonorrhoea may lead to psycho-social and physical complications and continued transmission. To improve diagnostic and treatment accuracy for gonorrhoea, the objective of this study was to examine epidemiological risk-factors for NAAT positive but culture negative cases.

Methods The study included all men and women in Stockholm having at least one positive gonorrhoea NAAT test with follow-up cultures taken during the period January 1, 2011-June 30, 2012. The total number of eligible cases during this period was 938. Data on sex, age, mode of transmission, symptoms, Chlamydia trachomatis co-infection and NAAT lab method were collected. Outcome was defined as positive NAAT but negative follow-up culture. Descriptive statistics and cross-tabulations with chi-squared tests were performed.

Results In total, 19\% of NAAT positive cases had no positive cultures $(\mathrm{N}=174)$. Diagnostic certainty was greater among men than women. Ten-percent of men and $37 \%$ of women with positive NAAT had negative cultures. Three laboratory NAAT methods were used with differences in subsequent negative culture proportions found among these methods.

Conclusion Women have an increased risk for incorrect diagnosis and/or treatment of gonorrhoea. Improved gonorrhoea testing practises are necessary to avoid systematic misdiagnoses and inappropriate treatments.

\section{P3.286 WITHDRAWN BY AUTHOR}

\section{P3.287 COMPARISON OF ANTIMICROBIAL SUSCEPTIBILITY OF NEISSERIA GONORRHOEAE ISOLATES OBTAINED FROM THE PHARYNX, RECTUM AND URETHRA IN MEN WHO HAVE SEX WITH MEN}

\section{doi:10.1136/sextrans-2013-051184.0742}

S Kidd, ${ }^{2} \mathrm{~L}$ Asbel, ${ }^{3} \mathrm{~T}$ Baldwin, ${ }^{4} \mathrm{~B}$ Gratzer, ${ }^{5} \mathrm{R}$ P Kerani, ${ }^{6} \mathrm{P}$ Pathela, ${ }^{1} \mathrm{~K}$ Pettus, ${ }^{7} \mathrm{O} 0$ Soge, ${ }^{8} \mathrm{~A}$ Stirland, 'H Weinstock. 'Centers for Disease Control and Prevention, Atlanta, GA, United States; ${ }^{2}$ Philadelphia Department of Public Health, Philadelphia, PA, United States;
${ }^{3}$ Texas Department of State Health Services, Laboratory Services Section, Austin, TX United States; ${ }^{4}$ Howard Brown Health Center, Chicago, IL, United States; ${ }^{5}$ University of Washington and Public Health - Seattle and King County, Seattle, WA, United States; ${ }^{6}$ New York City Department of Health and Mental Hygiene, New York City, NY, United States; 'Neisseria Reference Laboratory, Harborview Medical Center, Seattle, WA, United States; ${ }^{\circ}$ Los Angeles County Department of Public Health, Los Angeles, CA, United States

Background The emergence of cephalosporin resistance in Neisseria gonorrhoeae threatens gonorrhoea control programmes worldwide. Data on gonococcal antimicrobial susceptibility in the United States come from the Gonococcal Isolate Surveillance Project, which monitors susceptibility in male urethral isolates. Little is known about the susceptibility of isolates obtained from extra-genital sites. We sought to describe and compare antimicrobial susceptibility patterns of pharyngeal, rectal, and urethral gonococcal isolates obtained from men who have sex with men (MSM) at selected sentinel surveillance sites.

Methods We assessed the antimicrobial susceptibility of pharyngeal, rectal, and urethral gonococcal isolates collected from MSM at five sexually transmitted disease clinics throughout the United States. Minimum inhibitory concentrations (MICs) were determined by agar dilution at two regional laboratories, and elevated MICs were confirmed at the Centers for Disease Control and Prevention.

Results During December 2011-August 2012, a total of 85 pharyngeal, 99 rectal, and 315 urethral isolates from MSM were submitted. The proportion of isolates with an elevated cephalosporin or azithromycin MIC did not significantly differ by anatomic site: $1.2 \%$ of pharyngeal, $3.0 \%$ of rectal, and $3.2 \%$ of urethral isolates had an elevated cefixime MIC $(\geq 0.25 \mu \mathrm{g} / \mathrm{mL})(\mathrm{p}=0.79) ; 5.9 \%$ of pharyngeal, $7.1 \%$ of rectal, and $8.3 \%$ of urethral isolates had an elevated cefpodoxime MIC $(\geq 0.25 \mu \mathrm{g} / \mathrm{mL})(p=0.86) ; 1.2 \%$ of pharyngeal, $2.0 \%$ of rectal, and $4.1 \%$ of urethral isolates had an elevated ceftriaxone MIC $(\geq 0.125 \mu \mathrm{g} / \mathrm{mL})(p=0.47)$; and $2.4 \%$ of pharyngeal, $1.0 \%$ of rectal, and $1.6 \%$ of urethral isolates had an elevated azithromycin $\operatorname{MIC}(\geq 2.0 \mu \mathrm{g} / \mathrm{mL})(\mathrm{p}=0.91)$.

Conclusion Among MSM, the proportion of urethral isolates with an elevated cephalosporin or azithromycin MIC was similar to that of pharyngeal and rectal isolates. These findings suggest that, at the population level, gonococcal antimicrobial susceptibility surveillance based on urethral isolates from MSM adequately represents antimicrobial susceptibility of $N$. gonorrhoeae circulating among MSM.

\section{P3.288 ANTIMICROBIAL SUSCEPTIBILITY AND MOLECULAR EPIDEMIOLOGIC CLUSTERS OF NEISSERIA GONORRHOEAE STRAINS IN 2007 AND 2012 IN NANJING, CHINA}

doi:10.1136/sextrans-2013-051184.0743

${ }^{1}$ S Chen, ${ }^{1} Y$ Yin, ${ }^{1} X$ Chen, ${ }^{1} X$ Dai, ${ }^{1} R$ Yu, ${ }^{1} Y$ Han, ${ }^{2} \mathrm{M}$ Unemo. ${ }^{1}$ National Center for STD Control, China CDC, and the Chinese Academy of Medical Sciences \& Peking Uni, Nanjing, China; ${ }^{2}$ WHO Collaborating Centre for Gonorrhoea and Other STIs, Department of Laboratory Medicine, Microbiology, Örebro University Hospital, Örebro, Sweden

Background Gonorrhoea is the most prevalent bacterial sexually transmitted infection globally. It is of grave concern that Neisseria gonorrhoeae has developed resistance to mainly all antimicrobials introduced for treatment. China is located in the WHO Western Pacific Region (WPR), where most gonococcal antimicrobial resistance (AMR) has originated. However, the information regarding $\mathrm{AMR}$ and particularly molecular epidemiology of $N$. gonorrhoeae strains in China is highly limited. This study investigated the AMR and molecular epidemiologic clusters of N. gonorrhoeae in 2007 and 2012 in Nanjing, China.

Methods A total of 204 and 82 N. gonorrhoeae isolates were collected in 2007 and 2012, respectively, in Nanjing, China. The 